\title{
Acute kidney injury in hospitalized COVID-19 patients in Iran; a systematic review and meta-analysis
}

\author{
Alireza Saghafi ${ }^{1}$, Mohammad Aghaali $^{\circledR \oplus}$, Hossein Saghafi $^{3^{*}(\mathbb{D}}$ \\ ${ }^{1}$ School of Medicine, Qom University of Medical Sciences, Qom, Iran \\ ${ }^{2}$ Department of Social Medicine, School of Medicine, Qom University of Medical Sciences, Qom, Iran \\ ${ }^{3}$ Department of Internal Medicine, School of Medicine, Qom University of Medical Sciences, Qom, Iran
}

\section{A R T I C L E I N F O}

Article Type:

Review

Article History:

Received: 8 January 2021

Accepted: 1 February 2021

Published online: 3 February 2021

Keywords:

Acute kidney injury

COVID-19

Iran

Prevalence

Ethnicity

Meta-analysis

\begin{abstract}
A B S T R A C T
Acute kidney injury (AKI) is one of the complications in COVID-19 patients, which is reported with widely varied incidence rates in different studies and is known to have a major impact on prognosis and outcome of the disease. It is noticed that there are considerable differences in AKI rates between different countries. Rates in China are generally much lower than in Western Europe and the United States. One of the potential explanations is heterogeneity along racial and ethnic lines. This study aims to systematically investigate the scientific resources regarding AKI prevalence among hospitalized COVID-19 patients in Iran, and run a meta-analysis on currently published data. Web of Science, PubMed, Embase, Scopus, and Google Scholar databases were searched to identify the articles discussing the occurrence of AKI in hospitalized patients with COVID-19 in Iran. All observational and interventional studies with English full-text providing necessary data for analysis were included with no limitation in time of release or peer-review. Around, 4069 confirmed cases (age; 10-94) from 22 studies were included in the pooled outcome measurement. The proportion of hospitalized patients with COVID-19 in Iran who developed AKI was 24\% (95\% CI: 17-31\%). To the best of our knowledge, this is the first systematic review and meta-analysis to measure the prevalence of AKI in hospitalized COVID-19 patients in Iran. The geographical dissimilarities in the proportion of AKI among COVID-19 patients suggest a role for ethnical and racial differences in the tendency to develop renal involvement.
\end{abstract}

\section{Implication for health policy/practice/research/medical education}

In this systematic review and meta-analysis, we showed that $24 \%$ of hospitalized COVID-19 patients in Iran developed acute kidney injury. The geographical dissimilarities in the proportion of acute kidney injury among COVID-19 patients suggest a role for ethnical and racial differences in the tendency to develop renal involvement.

Please cite this paper as: Saghafi A, Aghaali M, Saghafi H. Acute kidney injury in hospitalized COVID-19 patients in Iran; a systematic review and meta-analysis. J Renal Inj Prev. 2021; 10(2): 09. doi: 10.34172/jrip.2021.09.

\section{Introduction}

On 31 December 2019, 27 cases of pneumonia of unknown etiology were identified in Wuhan city, Hubei province in China. These patients most notably presented with clinical symptoms of dry cough, dyspnea, fever, and bilateral lung infiltrates on imaging. The causative agent was identified from throat swab samples conducted by the Chinese Centre for Disease Control and Prevention (CCDC) on January 7, 2020, and was subsequently named severe acute respiratory syndrome coronavirus 2 (SARSCoV-2). The disease was named COVID-19 by the World Health Organization (WHO) (1). On March 11, 2020, the COVID-19 situation was characterized as a pandemic by WHO (2). Although the majority of patients presented with mild symptoms so far, a substantial number of them experienced fatal complications like various end organ failures, septic shock, pulmonary edema, and acute respiratory distress syndrome (ARDS). Acute kidney injury (AKI) is one of the complications in COVID-19 patients, which is reported with widely varied incidence rates in different studies and is known to have a major impact on prognosis and outcome of the disease (3-10). In a study in Wuhan City, China, during the first few months of the outbreak, the incidence of AKI and aggravation of chronic kidney disease (CKD) among hospitalized COVID-19 patients was concluded to be uncommon. No patient in the study developed AKI based on the diagnostic criteria, reportedly (3). However, the results of 
other studies support the probability of renal involvement in COVID-19. A study from China reported a $0.5 \%$ AKI incidence rate (5), while another study from New York City, the United States, calculated AKI development in hospitalized COVID-19 patients to be $36.6 \%$ (10). Understanding the pathophysiology of COVID-19 associated AKI is evolving, with no determined theory to date. It is suggested that the cause of kidney involvement might be multifactorial, with cardiovascular comorbidity and predisposing factors (e.g., sepsis, hypovolemia, and nephrotoxins) as important contributors (11). One study described the incidence of AKI as a feature of a thrombotic microangiopathy (TMA)-like phenomenon, secondary to SARS-CoV-2 infection (12). Moreover, virus particles were reported to be present in renal endothelial cells, indicating viremia as a possible cause of endothelial damage in the kidney and a probable contributor to AKI. Other possible mechanisms of injury are direct infection of the renal tubular epithelium and podocytes through an angiotensin-converting enzyme 2 (ACE2)-dependent pathway by SARS-CoV-2 which can cause mitochondrial dysfunction, acute tubular necrosis, the formation of protein reabsorption vacuoles, collapsing glomerulopathy, and protein leakage in Bowman's capsule (11). It is understood that complication of COVID-19 with AKI is associated with significantly higher in-hospital death rate (9), respiratory failure, and need for invasive mechanical ventilation (10); and thus is considered a marker of disease severity and a negative prognostic factor for survival (11). A hypothesis even suggests the possibility of CKD development in COVID-19 survivors complicated with AKI during the course of the disease (13). The difference in AKI prevalence between studies reporting hospitalized COVID-19 patients might partly be explained by different hospital admission policies applied to healthcare systems, and the quality of hospital care (14). However, that is unable to rationalize the substantial variation in AKI occurrence rate among COVID-19 patients ranging from $0 \%$ to $46 \%(3,15)$. It is noticed that there are considerable differences in AKI rates between different countries. Rates in China are generally much lower than in Western Europe and United States $(14,15)$. One of the potential explanations is heterogeneity along racial and ethnic lines (15). To the best of our knowledge, no publication discussed the issue independently to date, and hence, no validated analysis confirmed the role of ethnicity. This study aims to systematically investigate the scientific resources regarding AKI prevalence among hospitalized COVID-19 patients in Iran, and run a meta-analysis on currently published data. To date, this is the first metaanalysis study specifically concerned with the matter of race and hospital care by limiting the data inclusion to records from Iran. Results of this study can hint at the possible role of diversities in race and ethnicity and trigger further research in AKI predisposing factors, specifically in COVID-19 patients.

\section{Methods}

\section{Search strategy}

This analysis was conducted in line with the Preferred Reporting Items for Systematic Reviews and MetaAnalyses (PRISMA) guidelines (16). Web of Science, PubMed, Embase, Scopus, and Google Scholar databases were searched to identify the articles discussing the occurrence of AKI in hospitalized patients with COVID-19 in Iran. The whole process of identification was conducted in December 2020. The search terms were selected through the MeSH terms evaluation by two authors independently. Boolean operators were used for an advanced search unless not applicable. The main search string was as follows with minor differences applied to each of the search engines: ("COVID-19" OR "SARSCOV-2” OR “2019-nCoV” OR “coronavirus”) AND ("acute kidney injury" OR "acute renal injury" OR "acute kidney failure” OR "acute renal failure” OR "Oliguria” OR "elevated creatinine" OR "elevated serum creatinine" OR "elevated BUN" OR "elevated serum BUN" OR "Reduced GFR" OR "Reduced glomerular filtration rate") AND ("Iran" OR "name of prominent Iranian provinces and cities"). Further details and exact search query for each database are provided in Supplementary file 1.

\section{Inclusion and exclusion criteria}

All observational and interventional studies with English full-text providing necessary data for analysis were included with no limitation in time of release or peerreview. Exclusion of the articles based on each of the following criteria was conducted; review articles and other types of publications with no patient enrollment, case reports and case series with less than 10 patients, studies with data from outside Iran or out-patient population, studies limited to COVID-19 patients with renal presentation, articles which specifically focus on pediatric population, pregnant women, end-stage renal disease or chronic dialysis patients, and articles with insufficient data on AKI occurrence.

\section{Data extraction}

Search results were evaluated by two independent authors (AS and HS) and study selection was performed assessing the title and the abstract. Among nominated publications, articles' full-texts were reviewed to judge the eligibility for inclusion. A third reviewer (MA) decided on to resolve the disagreements at any phase. The following information was extracted from the studies using an organized checklist: name of the first author, date of the publication, name of the journal, study location, study design, peer-review status, the definition of AKI, type of population (general or special), type of admission ward (general or intensive care), sample size, age range, gender ratio, and proportion of AKI occurrence. General information of the included articles came under scrutiny to prevent duplication and data overlap. 


\section{Statistical analysis}

We used the random-effects model for investigating pooled prevalence with their specific 95\% confidence intervals (CIs). Study characteristics and estimated total proportion were graphically displayed by forest plots. To dissipate any heterogeneity, subgroup analyses with a random-effects model were carried out. Heterogeneity was measured using Cochran's $\mathrm{Q}$ test and $\mathrm{I}^{2}$ method. Publication bias was evaluated using funnel plot and egger's test. The effect of each of the studies on the total proportion and confidence interval was assessed by cumulative graph. A meta-regression was performed to determine the impact of moderator variables on the percentage of AKI occurrence in hospitalized patients with COVID-19. Stata 16.0 (StataCorp, College Station, TX, USA) was used for statistical analysis. $P<0.05$ was considered statistically significant.

\section{Results}

A total of 948 articles were identified through the systematic search of the databases as previously explained. After removal of all duplications, 746 records remained and were carefully gone through, which returned 125 results. Evaluation of the full-texts to assess eligibility was done and 100 publications were excluded due to either not fulfilling the inclusion criteria or being subject to any of the exclusion criteria. From 25 remained articles, 3 were excluded due to data overlap. Finally, 22 studies (1738) were selected and included in the review and metaanalysis. The search process and results are outlined in Figure 1.

The summary of characteristics extracted from studies is presented in Table 1. From 22 included studies, 9 were cohort $(17,19-21,23-25,33,36), 8$ were cross-sectional $(26,30-32,34,35,37,38)$, one was case-control (28), and 4 were trials $(18,22,27,29)$. Nineteen studies had completed peer-review processes and were published from June to November 2020; while three studies $(21,24,32)$ were not granted publication and peer-review at the time of inclusion. The pooled patient enrollment period was from $30 / 1 / 2020$ to $30 / 6 / 2020$. The studies provided a perfect geographical distribution. While 12 articles reported results from more than 7 hospitals located in various districts of Tehran, Iran's capital and the most populous city $(17-20,23,26,27,30,33,34,36,37)$, other studies were carried out in Zahedan (the Southeast) $(21,35)$, Abadan (the Southwest) (22), Qom (the center) (24), Urmia and Tabriz (the northwest) $(25,29,31)$, Mazandaran and Rasht (the north) $(28,38)$, and Nahavand (the west) (32). Three studies were conducted in a multi-center fashion $(21,25,30)$,
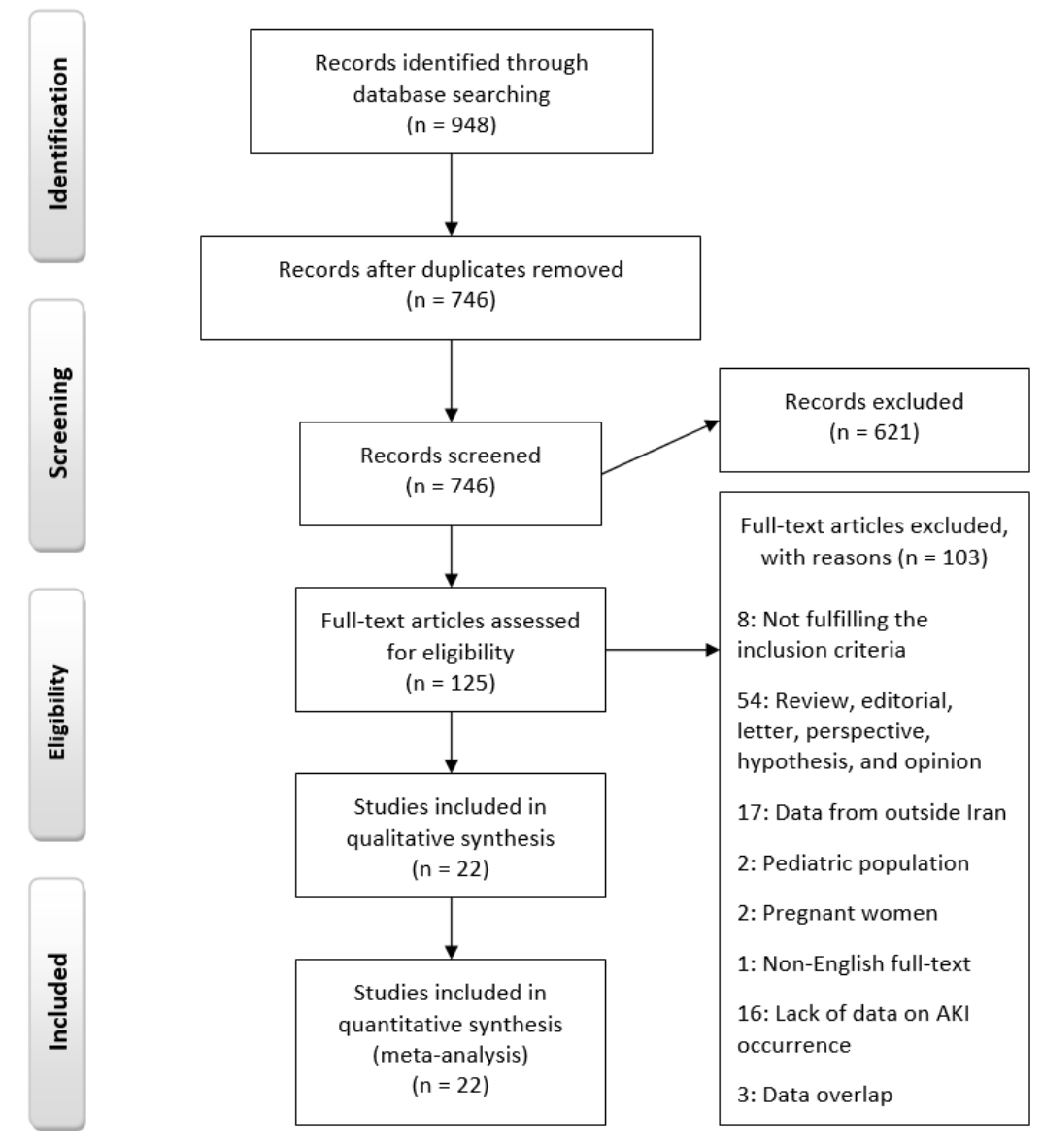

Figure 1. Flow diagram of studies. 
Table 1. Summary of study characteristics

\begin{tabular}{|c|c|c|c|c|c|c|c|c|c|c|c|}
\hline First Author & $\begin{array}{l}\text { Publication } \\
\text { date }\end{array}$ & Journal & $\begin{array}{l}\text { Hospital } \\
\text { City or province } \\
\text { Study Date }\end{array}$ & Study design & P-R & Aki Definition & Population & Sample Size & Age (y) & $\begin{array}{l}\text { Sex (female/ } \\
\text { male) }\end{array}$ & AKI \\
\hline \multirow{3}{*}{ Aghaaliakbari F } & \multirow{3}{*}{ Nov 2020} & \multirow{3}{*}{ Iran J Kidney Dis } & \multirow{3}{*}{$\begin{array}{l}\text { Firoozgar Tehran, } \\
1 / 3 \text { to } 30 / 6\end{array}$} & \multirow{3}{*}{$\begin{array}{l}\text { Retrospective } \\
\text { Cohort }\end{array}$} & \multirow{3}{*}{$\mathrm{Y}$} & \multirow{3}{*}{ KDIGO } & General & 617 & $18-94(58.49 \pm 15.8)$ & $255 / 362$ & $55 / 617(8.9 \%)$ \\
\hline & & & & & & & $\mathrm{DM}$ & 153 & $(64.4 \pm 11.7)$ & $62 / 91$ & $19 / 153(12.4 \%)$ \\
\hline & & & & & & & Non-DM & 464 & $(56.5 \pm 16.5)$ & $193 / 271$ & $36 / 464(7.8 \%)$ \\
\hline Rahmani H & Nov 2020 & $\begin{array}{l}\text { Int } \\
\text { Immunopharmacol }\end{array}$ & $\begin{array}{l}\text { Imam Khomeini, Tehran, } \\
20 / 4 \text { to } 20 / 5\end{array}$ & RCT & Y & & General & 66 & $47-73(60)$ & $27 / 39$ & $7 / 66(10.6 \%)$ \\
\hline Alamdari NM & Oct 2020 & $\begin{array}{l}\text { Diabetes Metab } \\
\text { Syndr }\end{array}$ & $\begin{array}{l}\text { Shahid Modarres,Tehran, } \\
1 / 4 \text { to } 3 / 5\end{array}$ & $\begin{array}{l}\text { Retrospective } \\
\text { Cohort }\end{array}$ & Y & Serum $\mathrm{Cr}$ & ICU & 157 & $63-72(67.58)$ & $19 / 138$ & $72 / 157(45.9 \%)$ \\
\hline \multirow{3}{*}{ Soleimani A } & \multirow{3}{*}{ Sep 2020} & \multirow{3}{*}{ Am J Hypertens } & \multirow{3}{*}{ Sina, Tehran, $20 / 2$ to $29 / 5$} & \multirow{3}{*}{$\begin{array}{l}\text { Retrospective } \\
\text { Cohort }\end{array}$} & \multirow{3}{*}{ Y } & \multirow{3}{*}{ KDIGO } & General & 636 & $(57.2 \pm 16.3)$ & $239 / 397$ & $74 / 636(11.6 \%)$ \\
\hline & & & & & & & HT & 254 & $(66.4 \pm 12.9)$ & $105 / 149$ & $49 / 254(19.3 \%)$ \\
\hline & & & & & & & Non-HT & 382 & $(51.2 \pm 15.5)$ & $134 / 248$ & $25 / 382(6.5 \%)$ \\
\hline $\begin{array}{l}\text { Hashemi-Shahri } \\
\text { SM }\end{array}$ & & & $\begin{array}{l}\text { MC ZAUMS, Zahedan } \\
29 / 2 \text { to } 31 / 4\end{array}$ & $\begin{array}{l}\text { Retrospective } \\
\text { Cohort }\end{array}$ & N & & General & 413 & $10-92(45.05 \pm 17.38)$ & $184 / 229$ & $20 / 413(4.9 \%)$ \\
\hline Eslami G & Aug 2020 & $\begin{array}{l}\text { J Antimicrob } \\
\text { Chemother }\end{array}$ & $\begin{array}{l}\text { Taleghani, Abadan } \\
18 / 3 \text { to } 16 / 4\end{array}$ & Open-label trial & Y & & General & 62 & $(62.5\{46-71\})$ & $31 / 31$ & $8 / 62(12.9 \%)$ \\
\hline Rahmani H & Aug 2020 & Daru & $\begin{array}{l}\text { Imam Khomeini, Tehran } \\
20 / 2 \text { to } 29 / 3\end{array}$ & $\begin{array}{l}\text { Prospective } \\
\text { Cohort }\end{array}$ & Y & KDIGO & General & 213 & $(60 \pm 14)$ & $100 / 113$ & $33 / 213$ (15.5\%) \\
\hline \multirow{3}{*}{ Hormati A } & & & \multirow{3}{*}{$\begin{array}{l}\text { Shahid Beheshti, Qom } \\
25 / 2 \text { to } 20 / 3\end{array}$} & \multirow{3}{*}{$\begin{array}{l}\text { Retrospective } \\
\text { Cohort }\end{array}$} & \multirow{3}{*}{ N } & \multirow{3}{*}{ KDIGO } & General & 200 & $(62.63 \pm 14.9)$ & $88 / 112$ & $67 / 200(33.5 \%)$ \\
\hline & & & & & & & Dead & 100 & $(66.4 \pm 14.8)$ & $43 / 57$ & $57 / 100(57 \%)$ \\
\hline & & & & & & & Dis & 100 & $(58.9 \pm 14.1)$ & $45 / 55$ & 10/100 (10\%) \\
\hline \multirow{3}{*}{$\begin{array}{l}\text { Mirza-Aghazadeh- } \\
\text { Attari M }\end{array}$} & \multirow{3}{*}{ Aug 2020} & \multirow{3}{*}{ Emerg Radiol } & \multirow{3}{*}{$\begin{array}{l}\text { MC, Urmia and/or Tabriz } \\
25 / 2 \text { to } 15 / 4\end{array}$} & \multirow{3}{*}{$\begin{array}{l}\text { Retrospective } \\
\text { Cohort }\end{array}$} & \multirow{3}{*}{ Y } & & General & 50 & $(65.4 \pm 16.77)$ & $23 / 27$ & $6 / 50(12 \%)$ \\
\hline & & & & & & & Dead & 23 & $(70.65 \pm 16.51)$ & $8 / 15$ & $6 / 23(26.1 \%)$ \\
\hline & & & & & & & Dis & 27 & $(60.93 \pm 15.94)$ & $15 / 12$ & $0 / 27(0 \%)$ \\
\hline \multirow{3}{*}{ Alamdari NM } & \multirow{3}{*}{ Sep 2020} & \multirow{3}{*}{ Tohoku J Exp Med } & Shahid Modarres & \multirow{3}{*}{ Cross-sectional } & \multirow{3}{*}{ Y } & & General & 459 & $(61.79 \pm 11.89)$ & $139 / 320$ & $128 / 459(27.9 \%)$ \\
\hline & & & Tehran & & & & Dead & 63 & $(70.58 \pm 10.66)$ & $14 / 49$ & $50 / 63(79.4 \%)$ \\
\hline & & & $30 / 1$ to $5 / 4$ & & & & Dis & 396 & $(60.39 \pm 11.47)$ & $125 / 271$ & 78/396 (19.7\%) \\
\hline $\begin{array}{l}\text { Davoudi-Monfared } \\
\text { E }\end{array}$ & Aug 2020 & $\begin{array}{l}\text { Antimicrob Agents } \\
\text { Chemother }\end{array}$ & $\begin{array}{l}\text { Imam Khomeini } \\
\text { Tehran } \\
29 / 2 \text { to } 3 / 4\end{array}$ & RCT & Y & KDIGO & General & 81 & $(57 \pm 15)$ & $37 / 44$ & $23 / 81(28.4 \%)$ \\
\hline Taghiloo S & Sep 2020 & J Med Virol & $\begin{array}{l}\text { Imam Khomeini } \\
\text { Mazandaran } \\
3 \text { to } 4\end{array}$ & Case-control & Y & & General & 61 & $(62\{50-72\})$ & $24 / 37$ & $6 / 61(9.8 \%)$ \\
\hline Valizadeh H & Oct 2020 & $\begin{array}{l}\text { Int } \\
\text { Immunopharmacol }\end{array}$ & $\begin{array}{l}\text { Imam Reza } \\
\text { Tabriz }\end{array}$ & RCT & Y & $\begin{array}{l}\text { Serum } \mathrm{Cr}> \\
1.5\end{array}$ & General & $39(40)$ & $19-69(52.35 \pm 8.15)$ & $9 / 31$ & $6 / 28(21.4 \%)$ \\
\hline
\end{tabular}


Table 1. Continued

\begin{tabular}{|c|c|c|c|c|c|c|c|c|c|c|c|}
\hline First Author & $\begin{array}{l}\text { Publication } \\
\text { date }\end{array}$ & Journal & $\begin{array}{l}\text { Hospital } \\
\text { City or province } \\
\text { Study Date } \\
\end{array}$ & Study design & P-R & Aki Definition & Population & Sample Size & Age (y) & $\begin{array}{l}\text { Sex (female/ } \\
\text { male) }\end{array}$ & AKI \\
\hline Jamaati H & Aug 2020 & $\begin{array}{l}\text { Biomedical and } \\
\text { Biotechnology } \\
\text { Research J }\end{array}$ & $\begin{array}{l}\text { MC SBMU, Tehran } \\
3 \text { to } 5\end{array}$ & Cross-sectional & Y & $\begin{array}{l}\text { Serum } \mathrm{Cr}> \\
1.2\end{array}$ & $\mathrm{ICU}$ & 36 & $26-86(57.9 \pm 16.8)$ & $8 / 28$ & $6 / 36(16.6 \%)$ \\
\hline Mahmoodpoor A & Jun 2020 & Anesth Pain Med & $\begin{array}{l}\text { Imam Reza, Tabriz } \\
20 / 2 \text { to } 20 / 4\end{array}$ & Cross-sectional & Y & & $\mathrm{ICU}$ & 200 & & & $50 / 200(25 \%)$ \\
\hline Mahmoudi H & & & $\begin{array}{l}\text { Alimoradiyan, Nahavand } \\
17 / 2 \text { to } 11 / 4\end{array}$ & Cross-sectional & $\mathrm{N}$ & $\begin{array}{l}\text { Elevation in } \\
\text { Serum } \mathrm{Cr} \text { and } \\
\text { Urea }\end{array}$ & General & 100 & $20-82(51)$ & $31 / 69$ & $35 / 100(35 \%)$ \\
\hline \multirow{3}{*}{ Mansour A } & \multirow{3}{*}{ Nov 2020} & \multirow{3}{*}{ EXCLI J } & Shariati & \multirow{3}{*}{$\begin{array}{l}\text { Retrospective } \\
\text { Cohort }\end{array}$} & \multirow{3}{*}{ Y } & \multirow{3}{*}{$\begin{array}{l}\text { Serum } \mathrm{Cr}> \\
1.5\end{array}$} & General & $339(353)$ & $18-97(61.67 \pm 16.38)$ & $150 / 203$ & 66/339 (19.5\%) \\
\hline & & & Tehran & & & & DM & $101(111)$ & $(63.66 \pm 13.32)$ & $49 / 62$ & $25 / 101(24.8 \%)$ \\
\hline & & & $25 / 2$ to $21 / 4$ & & & & Non-DM & $238(242)$ & $(60.76 \pm 17.56)$ & $101 / 141$ & $41 / 238(17.2 \%)$ \\
\hline Besharat S & Sep 2020 & $\begin{array}{l}\text { Open Access Maced J } \\
\text { Med Sci }\end{array}$ & $\begin{array}{l}\text { Modarres } \\
\text { Tehran } \\
1 / 4 \text { to } 30 / 4\end{array}$ & Cross-sectional & Y & & Dead & 62 & $28-95(67.62 \pm 15.07)$ & $18 / 44$ & $18 / 62(29 \%)$ \\
\hline \multirow{3}{*}{ Rokni M } & \multirow{3}{*}{ Nov 2020} & \multirow{3}{*}{ BMC Infect Dis } & Buali & \multirow{3}{*}{ Cross-sectional } & \multirow{3}{*}{ Y } & \multirow{3}{*}{$\begin{array}{l}\text { Serum } \mathrm{Cr}> \\
1.4\end{array}$} & General & $212(233)$ & (49.8) & $84 / 149$ & $47 / 212(22.2 \%)$ \\
\hline & & & Zahedan & & & & Dead & $23(28)$ & (65.3) & $8 / 20$ & $15 / 23(65.2 \%)$ \\
\hline & & & $29 / 2$ to $24 / 5$ & & & & Dis & $189(205)$ & (47.6) & $76 / 129$ & $32 / 189$ (16.9\%) \\
\hline Barkhordari K & Aug 2020 & J Card Surg & $\begin{array}{l}\text { THC } \\
\text { Tehran } \\
\text { 29/2 to } 10 / 4\end{array}$ & $\begin{array}{l}\text { Retrospective } \\
\text { Cohort }\end{array}$ & Y & KDIGO & $\begin{array}{l}\text { Concurrent } \\
\text { Cardiac } \\
\text { Surgery }\end{array}$ & 25 & $(57.33 \pm 15.13)$ & $7 / 18$ & $10 / 25(40 \%)$ \\
\hline \multirow{3}{*}{ Ghaffari Rahbar M } & \multirow{3}{*}{ Jul 2020} & \multirow{3}{*}{ Transpl Infect Dis } & $\begin{array}{l}\text { Labbafinejad } \\
\text { Tehran }\end{array}$ & \multirow{3}{*}{ Cross-sectional } & \multirow{3}{*}{$\mathrm{Y}$} & \multirow{3}{*}{$\begin{array}{l}>30 \% \\
\text { decrease in } \\
\text { eGFR (MDRD) }\end{array}$} & $\begin{array}{l}\text { Kidney } \\
\text { Transplant }\end{array}$ & 19 & $29-66(47.6 \pm 12.4)$ & $6 / 13$ & $14 / 19(73.7 \%)$ \\
\hline & & & $20 / 2$ to $15 / 4$ & & & & Dead & 9 & $(51.2 \pm 12.8)$ & $1 / 8$ & $7 / 9(77.8 \%)$ \\
\hline & & & & & & & Dis & 10 & $(44.3 \pm 11.8)$ & $5 / 5$ & $7 / 10$ (70\%) \\
\hline \multirow{3}{*}{ Monfared A } & \multirow{3}{*}{ Jul 2020} & \multirow{3}{*}{ Transpl Infect Dis } & Razi & \multirow{3}{*}{ Cross-sectional } & \multirow{3}{*}{ Y } & \multirow{3}{*}{ KDIGO } & $\begin{array}{l}\text { Kidney } \\
\text { Transplant }\end{array}$ & 22 & $(52\{40.75-62.75\})$ & $7 / 15$ & $12 / 22(54.5 \%)$ \\
\hline & & & $20 / 2$ to $19 / 4$ & & & & Dead & 6 & & & 6/6 (100\%) \\
\hline & & & & & & & Dis & 16 & & & $6 / 16(37.5 \%)$ \\
\hline
\end{tabular}

P-R: Peer-review, Y: Yes, N: No, MC: Multi-center, RCT: Randomized Clinical Trial, Cr: Creatinine, DM: Diabetes, HT: Hypertension, Dead: Deceased, Dis: Discharged.

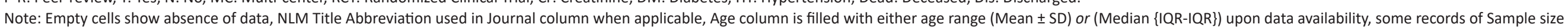
column are in a No.1 (No.2) fashion. No.2 shows the real sample size, while no.1 shows how many had normal baseline Cr. Patients with high baseline Cr were put aside in order not to be mistaken with AKI when required 
while the rest were single-center studies. 7 articles defined AKI according to KDIGO criteria $(17,20,23,24,27,36$, 38) which is an increase in serum creatinine by $0.3 \mathrm{mg} /$ $\mathrm{dL}$ within 48 hours or a $50 \%$ increase in serum creatinine from baseline within 7 days (39). One study outlined AKI as $>30 \%$ decrease in eGFR estimated by MDRD equation (37), while AKI was defined roughly by serum creatinine cut-off if specified in other studies. In three studies, patients were specifically from intensive care unit (ICU) $(19,30,31)$. Two studies enrolled a population of kidney transplant recipients $(37,38)$, one only deceased patients (34), and one patients who underwent concurrent cardiac surgery (36). The funnel plot to evaluate publication bias is demonstrated in Figure 2.

Around 4069 confirmed cases (age; $10-94$ ) were included in the pooled outcome measurement. The proportion of hospitalized patients with COVID-19 in Iran who developed AKI was 24\% (95\% CI: 17-31\%). Figure 3 shows the forest plot of AKI in COVID-19 hospitalized patients. The effect of each of the studies on the total proportion and confidence interval is displayed by cumulative graph (Figure 4). Subgroup analyses revealed AKI development to be higher in ICU than general ward (30\%, 95\% CI: $13-$ $47 \%$, versus $23 \%, 95 \%$ CI: $16-31 \%)$. Studies which defined AKI according to either KDIGO or decline in GFR showed higher AKI occurrence than ones with serum $\mathrm{Cr}$ as an indicator (32\%, 95\% CI: $16-47 \%$, versus $21 \%, 95 \%$ CI: $15-27 \%)$. Observational studies returned higher AKI percentage than trials $(26 \%, 95 \%$ CI: $17-34 \%$, versus $17 \%$, 95\% CI: $10-25 \%)$. None of the subgroup analysis results were, however, statistically significant $(P=0.52, P=0.20$ and $P=0.15$ respectively). There was no difference between peer-reviewed and not-peer-reviewed articles in terms of AKI proportion (24\%, 95\% CI: $17-32 \%$, versus 24\%, 95\% CI: 5-43\%). Figure 5 summarizes the outcomes of subgroup analysis.

Pooled data from deceased patients showed a significantly higher proportion of AKI compared with the patients who were discharged from hospital (62\%, 95\% CI:

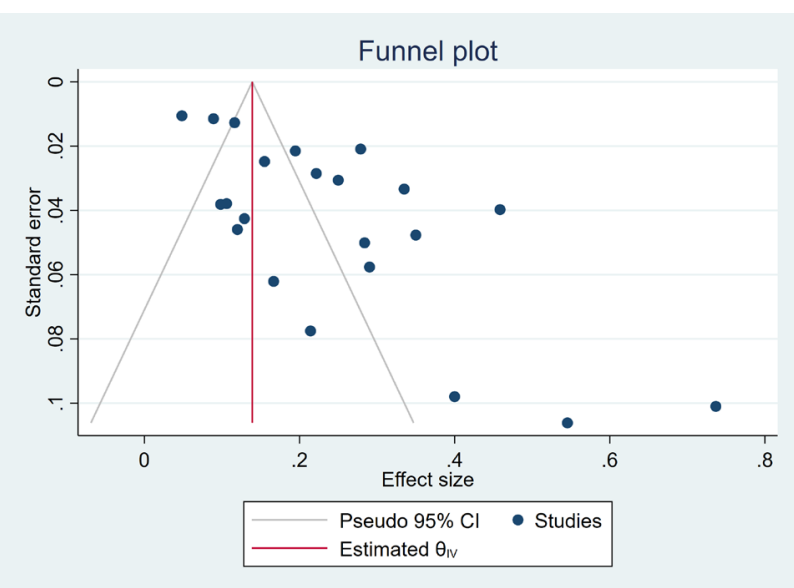

Figure 2. Funnel plot based on total studies.
42-82\%, versus $28 \%, 95 \%$ CI: 7-49\%, $P=0.02$ ) (Figure 6 and Figure 7). It should be taken into consideration that the majority of included studies did not report segregated data regarding survival. Hence, these percentages do not represent the whole proportion. AKI was found in 64\% (95\% CI: 46-83\%) of kidney transplant patients, data from two independent studies indicated (Figure 8).

Evaluating the variables in terms of their impact on AKI occurrence, it turned out that kidney transplantation and death outcome are the two predictors. Details are summarized in Table 2.

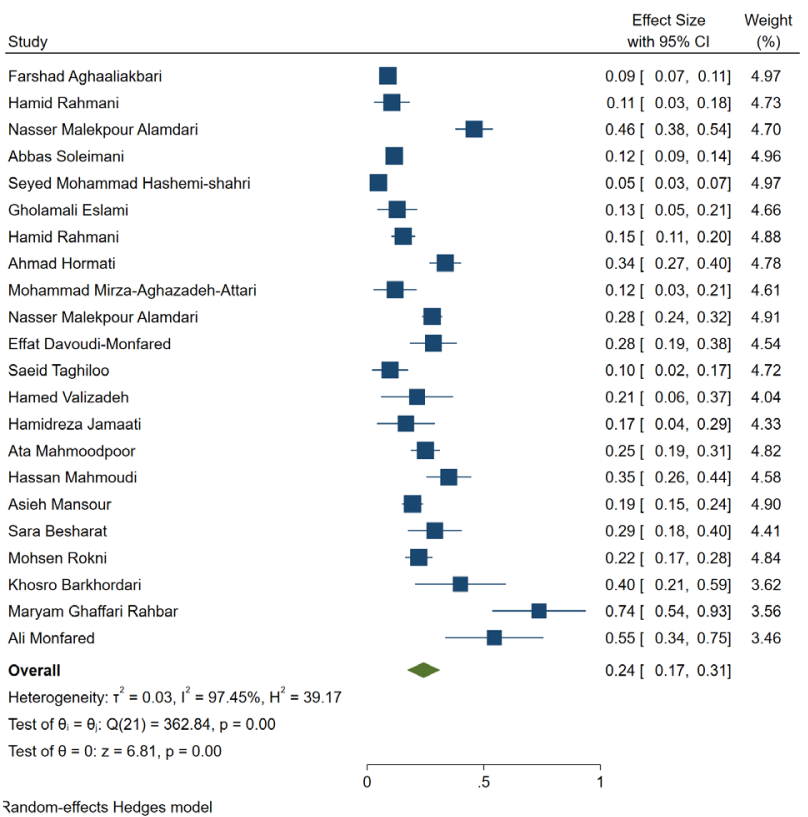

Figure 3. Forest plot for proportions of AKI. Note: Weights are from random-effects analysis.

\begin{tabular}{|c|c|c|c|c|c|c|}
\hline Study & & & & $\begin{array}{l}\text { ect Size } \\
95 \% \mathrm{Cl}\end{array}$ & P-value & Num \\
\hline Farshad Aghaaliakbari & - & & 0.09[ & $0.07,0.11]$ & 0.000 & 1 \\
\hline Hamid Rahmani & $\rightarrow$ & & 0.09[ & $0.07,0.11]$ & 0.000 & 2 \\
\hline Nasser Malekpour Alamdari & • & & 0.22[ & $-0.02,0.45]$ & 0.072 & 3 \\
\hline Abbas Soleimani & $\longrightarrow$ & & 0.19[ & $0.02,0.36]$ & 0.032 & 4 \\
\hline Seyed Mohammad Hashemi-shahri & $\cdot$ & & 0.16[ & $0.01,0.31]$ & 0.031 & 5 \\
\hline Gholamali Eslami & $\longrightarrow$ & & 0.16[ & $0.04,0.28]$ & 0.011 & 6 \\
\hline Hamid Rahmani & $\longrightarrow$ & & 0.16[ & $0.05,0.26]$ & 0.003 & 7 \\
\hline Ahmad Hormati & $\longrightarrow$ & & 0.18[ & $0.08,0.28]$ & 0.000 & 8 \\
\hline Mohammad Mirza-Aghazadeh-Attari & $\longrightarrow$ & & 0.17[ & $0.08,0.26]$ & 0.000 & 9 \\
\hline Nasser Malekpour Alamdari & $\longrightarrow$ & & 0.18[ & $0.10,0.26]$ & 0.000 & 10 \\
\hline Effat Davoudi-Monfared & $\longrightarrow$ & & 0.19[ & $0.12,0.27]$ & 0.000 & 11 \\
\hline Saeid Taghiloo & $\longrightarrow$ & & 0.18[ & $0.11,0.25]$ & 0.000 & 12 \\
\hline Hamed Valizadeh & - - & & 0.18[ & $0.12,0.25]$ & 0.000 & 13 \\
\hline Hamidreza Jamaati & $\because-$ & & 0.18[ & $0.12,0.24]$ & 0.000 & 14 \\
\hline Ata Mahmoodpoor & $\rightarrow$ & & 0.19[ & $0.13,0.24]$ & 0.000 & 15 \\
\hline Hassan Mahmoudi & $\rightarrow$ & & 0.20[ & $0.14,0.25]$ & 0.000 & 16 \\
\hline Asieh Mansour & - & & 0.20[ & $0.14,0.25]$ & 0.000 & 17 \\
\hline Sara Besharat & $\rightarrow$ & & 0.20[ & $0.15,0.25]$ & 0.000 & 18 \\
\hline Mohsen Rokni & $\rightarrow-$ & & 0.20[ & $0.15,0.25]$ & 0.000 & 19 \\
\hline Khosro Barkhordari & $\rightarrow-$ & & 0.21[ & $0.16,0.26]$ & 0.000 & 20 \\
\hline Maryam Ghaffari Rahbar & $\longrightarrow$ & & 0.23[ & $0.16,0.30]$ & 0.000 & 21 \\
\hline \multirow[t]{2}{*}{ Ali Monfared } & $\longrightarrow$ & & 0.24[ & $0.17,0.31]$ & 0.000 & 22 \\
\hline & .2 & .4 & 6 & & & \\
\hline
\end{tabular}

Figure 4. Cumulative graph for showing the effect of each study on the total proportion. 


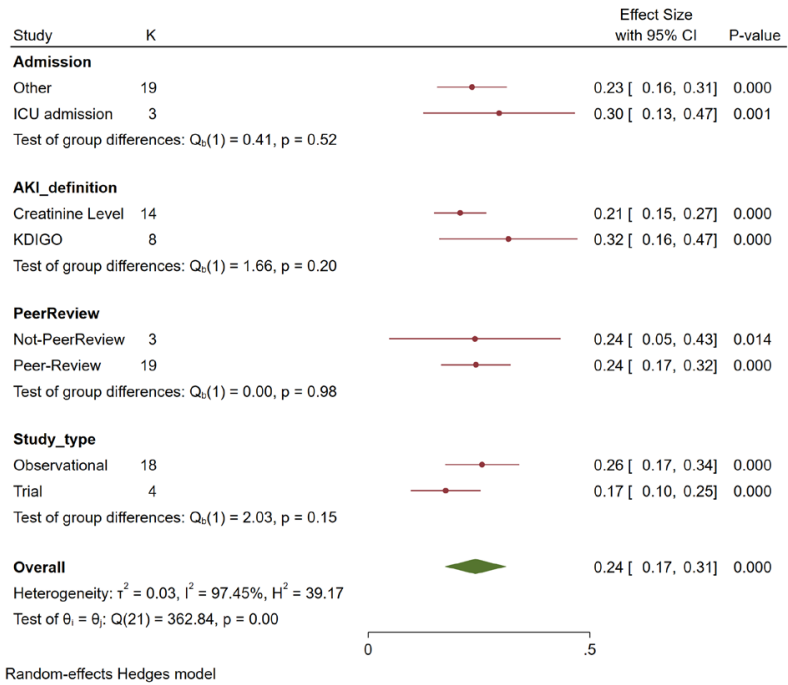

Figure 5. Summary of outcomes of subgroup analyses.

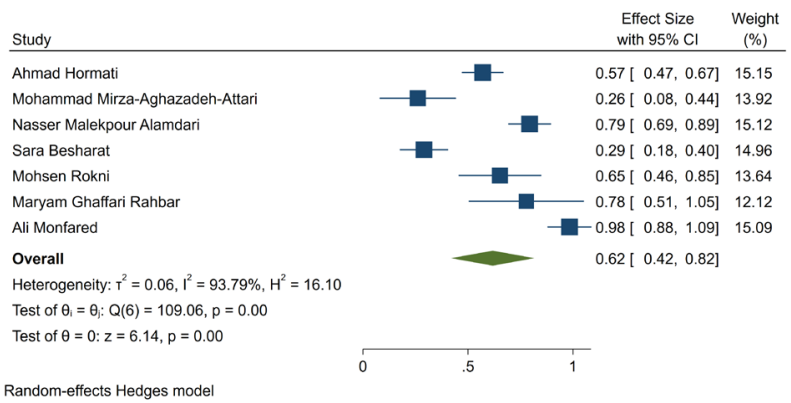

Figure 6. Forest plot for proportions of AKI in deceased patients.

\section{Discussion}

Since December 2019, when COVID-19 emerged in Wuhan city and rapidly spread throughout China, to date, when it still is the most prominent health issue throughout the world, the data on the disease's presentation, clinical course, and outcome was rapidly growing (5). Because of the novelty of the disease, our knowledge about it is subject to evolution, and the combination of reports yields a more comprehensive understanding as time goes by. We conducted a systematic review and metaanalysis on the studies which reported the occurrence of AKI in hospitalized COVID-19 patients in Iran. We found out that $24 \%$ of patients developed AKI, which

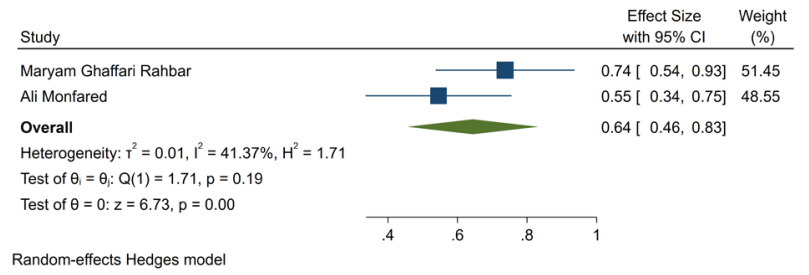

Figure 8. Forest plot for proportions of AKI in kidney transplant patients.

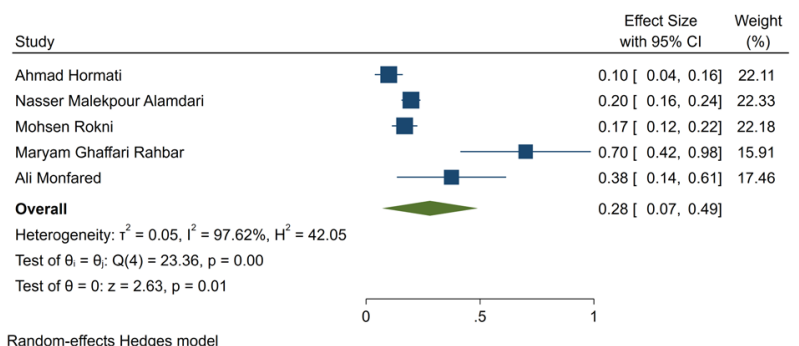

Figure 7. Forest plot for proportions of AKI in discharged patients.

indicated kidney damage to be a common complication of the disease. Compared to previous reports, our study returned a higher percentage of AKI. Two previously published meta-analyses, calculated the prevalence of AKI $4.5 \%$ (40), and $17 \%$ (41). The noteworthy point is that in those meta-analyses, the majority of data came from China, which had published most of the cited documents due to the precedence of disease emergence there. A primary report from china had even concluded that AKI is not a complication of COVID-19 (3), although the following information modified the presumption (14). However, several researchers noticed lower rates of AKI in reports from China than elsewhere $(10,15)$. It means that the conclusion of previous meta-analysis might have been affected by the Chinese reports, while to date, only about $0.1 \%$ of COVID- 19 cases were from China (42). Hirsch et al conducted a retrospective cohort study on admitted COVID-19 patients in the New York metropolitan area, which showed $36.6 \%$ AKI occurrence (10). Another multi-center observational study from New York reported AKI development in up to $46 \%$ of admitted COVID-19 patients (43). Although few European data are currently available on this topic, published data presented generally higher rates than Chinese studies (15). These

Table 2. Meta-regression details and results

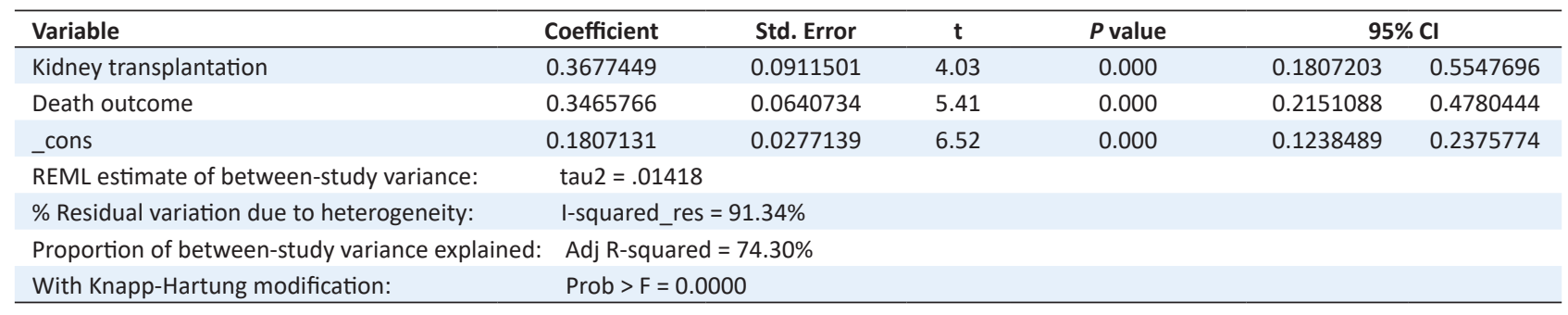


geographical differences can potentially be explained by the differences in admission criteria, hospital care, and ethnic predisposition to kidney involvement. Our study indicated the development of AKI in about a quarter of patients admitted in more than 18 hospitals in various regions of Iran, remarkably higher than most of the Chinese reports and close to reports from Western Europe and United States $(14,15)$.

In our study, ICU admitted patients had a higher rate of AKI development than general ward admissions (30\% versus 23\%). However, the difference was not statistically significant. Moreover, general AKI prevalence in ICU patients was close and even sometimes higher in previous reports from Iran (44-46). An explanation might be that although AKI is a common complication, it is not a key contributor to disease severity in COVID-19 patients.

In another subgroup study, we analyzed the data from studies which defined AKI either in line with KDIGO criteria (39) or GFR estimation and studies that either did not specify or used serum creatinine to assess AKI. The proportion was higher in the first group (32\% versus $21 \%$ ), although not statistically significant. It is possible that applying a more precise definition to AKI diagnosis leads to a higher occurrence rate.

We conducted an analysis to determine if there is a difference in AKI percentage in deceased compared to discharged patients. Seven articles had reported the necessary data, and analysis revealed that the prevalence of AKI was significantly higher in patients who did not survive than the ones who discharged (62\% versus $28 \%$, $P=0.02$ ). Richardson et al performed an observational study which returned $62.7 \%$ AKI percentage in deceased patients compared with $8.5 \%$ in discharged ones (4). However, due to the scarcity of data, it is yet to develop a conclusion.

Two studies that specifically included kidney transplant recipients who were admitted due to COVID-19 were analyzed. It was shown that $64 \%$ of patients developed AKI. AKI is a critical complication in kidney transplant patients, because of its possible contribution to allograft rejection.

Several variables were assessed to determine if they had an impact on AKI occurrence by meta-regression. Two predictors, death outcome and kidney transplantation, were revealed. We assume that the high percentage of AKI development in deceased patients might partly be contributed to multiple organ failure in the terminal phase of severe disease. Further research should be performed to evaluate the impact of AKI as a variable on the survival of COVID-19 patients, limiting confounders effect.

Our review faced some limitations. The data on COVID-19 patients was limited due to the novelty of the disease, and more data might influence the proportion. There was heterogeneity regarding type of study, definition of AKI, and study population. The quality of included studies was varied. Some of the included articles did not mention specific data on AKI occurrence. A serum creatinine cut-off was set to determine AKI in those reports.

\section{Conclusion}

To the best of our knowledge, this is the first systematic review and meta-analysis to measure the prevalence of AKI in hospitalized COVID-19 patients in Iran. We showed that $24 \%$ of patients developed AKI. The geographical dissimilarities in the proportion of AKI among COVID-19 patients suggest a role for ethnical and racial differences in the tendency to develop renal involvement. Further research is warranted to determine the significance of ethnicity, while other factors like the quality of hospital care might also play a role. COVID-19 needs a comprehensive clinical and research approach, and our understanding of this disease is still insufficient. Large-scale studies are recommended to unfold more accurate characteristics of COVID-19.

\section{Authors' contribution}

AS, MA, and HS contributed equally to prepare the study concept and design. AS and HS carried out the acquisition of data. MA performed the statistical analysis and interpreted data. AS wrote the primary manuscript. HS critically revised the manuscript. All authors read and signed the final manuscript.

\section{Conflicts of interest}

The authors declare no conflict of interest.

\section{Ethical considerations}

The study was approved by the ethics committee of Qom University of Medical Sciences (Code: IR.MUQ. REC.1399.226). Ethical issues (including plagiarism, data fabrication, double publication) have been completely observed by the authors.

\section{Funding/Support}

None.

\section{Supplementary files}

Online Supplementary file 1 shows search strategies used in this study.

\section{References}

1. Sohrabi C, Alsafi Z, O'Neill N, Khan M, Kerwan A, AlJabir A, et al. World Health Organization declares global emergency: A review of the 2019 novel coronavirus (COVID-19). Int J Surg. 2020;76:71-6. doi: 10.1016/j. ijsu.2020.02.034.

2. Bedford J, Enria D, Giesecke J, Heymann DL, Ihekweazu C, Kobinger G, et al. COVID-19: towards controlling of a pandemic. Lancet. 2020;395:1015-8. doi: 10.1016/S01406736(20)30673-5. 
3. Wang L, Li X, Chen H, Yan S, Li D, Li Y, et al. Coronavirus disease 19 infection does not result in acute kidney injury: An analysis of 116 hospitalized patients from Wuhan, China. Am J Nephrol. 2020;51:343-8. doi: 10.1159/000507471.

4. Richardson S, Hirsch JS, Narasimhan M, Crawford JM, McGinn T, Davidson KW, et al. Presenting Characteristics, Comorbidities, and Outcomes among 5700 Patients Hospitalized with COVID-19 in the New York City Area. JAMA. 2020;323:2052-9. doi: 10.1001/jama.2020.6775.

5. Guan W, Ni Z, Hu Y, Liang W, Ou C, He J, et al. Clinical characteristics of coronavirus disease 2019 in China. N Engl J Med. 2020;382:1708-20. doi: 10.1056/NEJMoa2002032.

6. Huang C, Wang Y, Li X, Ren L, Zhao J, Hu Y, et al. Clinical features of patients infected with 2019 novel coronavirus in Wuhan, China. Lancet. 2020;395:497-506. doi: 10.1016/ S0140-6736(20)30183-5.

7. Zhou F, Yu T, Du R, Fan G, Liu Y, Liu Z, et al. Clinical course and risk factors for mortality of adult inpatients with COVID-19 in Wuhan, China: a retrospective cohort study. Lancet. 2020;395:1054-62. doi: 10.1016/S01406736(20)30566-3.

8. Chen G, Wu D, Guo W, Cao Y, Huang D, Wang H, et al. Clinical and immunological features of severe and moderate coronavirus disease 2019. J Clin Invest. 2020;130:2620-9. doi: $10.1172 /$ JCI137244.

9. Cheng Y, Luo R, Wang K, Zhang M, Wang Z, Dong L, et al. Kidney disease is associated with in-hospital death of patients with COVID-19. Kidney Int. 2020;97:829-38. doi: 10.1016/j.kint.2020.03.005.

10. Hirsch JS, Ng JH, Ross DW, Sharma P, Shah HH, Barnett $\mathrm{RL}$, et al. Acute kidney injury in patients hospitalized with COVID-19. Kidney Int. 2020;98:209-18. doi: 10.1016/j. kint.2020.05.006.

11. Ronco C, Reis T, Husain-Syed F. Management of acute kidney injury in patients with COVID-19. Lancet Respir Med. 2020;8:738-42. doi: 10.1016/S2213-2600(20)30229-0.

12. Henry BM, Benoit SW, de Oliveira MHS, Lippi G, Favaloro EJ, Benoit JL. ADAMTS13 activity to von Willebrand factor antigen ratio predicts acute kidney injury in patients with COVID-19: Evidence of SARS-CoV-2 induced secondary thrombotic microangiopathy. Int J Lab Hematol. 2020. doi: 10.1111/ijlh.13415.

13. Khoshdel-Rad N, Zahmatkesh E, Shpichka A, Timashev P, Vosough M. Outbreak of chronic renal failure: will this be a delayed heritage of COVID-19? J Nephrol. 2020. doi: 10.1007/s40620-020-00851-9.

14. Zheng X, Zhao Y, Yang L. Acute kidney injury in COVID-19: the chinese experience. Semin Nephrol. 2020;40:430-42. doi: 10.1016/j.semnephrol.2020.09.001.

15. Farouk SS, Fiaccadori E, Cravedi P, Campbell KN. COVID-19 and the kidney: what we think we know so far and what we don't. J Nephrol. 2020;33:1213-8. doi: 10.1007/ s40620-020-00789-y.

16. Moher D, Liberati A, Tetzlaff J, Altman DG, Altman D, Antes $G$, et al. Preferred reporting items for systematic reviews and meta-analyses: The PRISMA statement. PLoS Med. 2009;6:e1000097. doi: 10.1371/journal.pmed.1000097.

17. Aghaaliakbari F, Abbasi MA, Ranjbar M, Makiani MJ, Farrokhpour M, Tameshkel FS, et al. Angiotensin converting enzyme inhibitors, a risk factor of poor outcome in diabetic patients with COVID-19 infection. Iran J Kidney
Dis. 2020;14:482-7.

18. Rahmani H, Davoudi-Monfared E, Nourian A, Khalili $\mathrm{H}$, Hajizadeh $\mathrm{N}$, Jalalabadi NZ, et al. Interferon $\beta-1 \mathrm{~b}$ in treatment of severe COVID-19: A randomized clinical trial. Int Immunopharmacol. 2020;88:106903. doi: 10.1016/j. intimp.2020.106903.

19. Alamdari NM, Rahimi FS, Afaghi S, Zarghi A, Qaderi S, Tarki FE, et al. The impact of metabolic syndrome on morbidity and mortality among intensive care unit admitted COVID-19 patients. Diabetes Metab Syndr. 2020;14:197986. doi: $10.1016 /$ j.dsx.2020.10.012.

20. Soleimani A, Kazemian S, Karbalai Saleh S, Aminorroaya A, Shajari Z, Hadadi A, et al. Effects of Angiotensin Receptor Blockers (ARBs) on In-Hospital Outcomes of Patients With Hypertension and Confirmed or Clinically Suspected COVID-19. Am J Hypertens. 2020;33:1102-11. doi: $10.1093 /$ ajh/hpaa149.

21. Seyed Mohammad HS, Seyed Mohammad Nasiraldin T, Alireza AM, Mahdi M, Hassan OA, Seyed Mehdi T, et al. Epidemiological and Clinical Risk Factors Related to Severe COVID-19 in Iran: A multi-center study. Res Sq. 2021. doi: 10.21203/rs.3.rs-105648/v2.

22. Eslami G, Mousaviasl S, Radmanesh E, Jelvay S, Bitaraf S, Simmons B, et al. The impact of sofosbuvir/daclatasvir or ribavirin in patients with severe COVID-19. J Antimicrob Chemother. 2020;75:3366-72. doi: 10.1093/jac/dkaa331.

23. Rahmani H, Davoudi-Monfared E, Nourian A, Nabiee M, Sadeghi S, Khalili H, et al. Comparing outcomes of hospitalized patients with moderate and severe COVID-19 following treatment with hydroxychloroquine plus atazanavir/ritonavir. Daru. 2020;28:625-34. doi: 10.1007/ s40199-020-00369-2.

24. Ahmad H, SeyedYaser Foroghi G, masoudreza s, Ali G, Saeede J, Amir J, et al. Clinical characteristics and Mortality risk factors among COVID-19 patients in Qom-Iran; The results of a Retrospective Cohort study. Res Sq. 2021. doi: 10.21203/rs.3.rs-42497/v1.

25. Mirza-Aghazadeh-Attari M, Zarrintan A, Nezami N, Mohammadi A, Zarrintan A, Mohebbi I, et al. Predictors of coronavirus disease 19 (COVID-19) pneumonitis outcome based on computed tomography (CT) imaging obtained prior to hospitalization: a retrospective study. Emerg Radiol. 2020;27:653-61. doi: 10.1007/s10140-020-01833-x.

26. Alamdari NM, Afaghi S, Rahimi FS, Tarki FE, Tavana S, Zali A, et al. Mortality risk factors among hospitalized COVID-19 patients in a major referral center in Iran. Tohoku J Exp Med. 2020;252:73-84. doi: 10.1620/ tjem.252.73.

27. Davoudi-MonfaredE, RahmaniH, KhaliliH,Hajiabdolbaghi M, Salehi M, Abbasian L, et al. A randomized clinical trial of the efficacy and safety of interferon $\beta-1 \mathrm{a}$ in treatment of severe COVID-19. Antimicrob Agents Chemother. 2020;64:e01061-20. doi: 10.1128/AAC.01061-20.

28. Taghiloo S, Aliyali M, Abedi S, Mehravaran H, Sharifpour A, Zaboli E, et al. Apoptosis and immunophenotyping of peripheral blood lymphocytes in Iranian COVID-19 patients: Clinical and laboratory characteristics. J Med Virol. 2020. doi: 10.1002/jmv.26505.

29. Valizadeh H, Abdolmohammadi-vahid S, Danshina S, Ziya Gencer M, Ammari A, Sadeghi A, et al. Nano-curcumin therapy, a promising method in modulating inflammatory 
cytokines in COVID-19 patients. Int Immunopharmacol. 2020;89:107088. doi: 10.1016/j.intimp.2020.107088.

30. Jamaati H, Fadaizadeh L, Khoundabi B, Hashemian S, Monjazabi F, Jahangirifard A, et al. COVID-19-Related severe heterogeneous acute respiratory distress syndrome: A therapeutic challenge. Biomed Biotechnol Res J. 2020;4:S75-82. doi: 10.4103/bbrj.bbrj_133_20.

31. Mahmoodpoor A, Shadvar K, Ghamari AA, Lameh MM, Ardebili RA, Hamidi M, et al. Management of critically ill patients with covid-19: What we learned and what we do. Anesth Pain Med. 2020;10:1-4. doi: 10.5812/aapm.104900.

32. Hassan M, Mohammad Yousef A, Narges Mofrad T, Alireza B. Res Sq. 2021. doi: 10.21203/rs.3.rs-25164/v1.

33. Mansour A, Sajjadi-Jazi SM, Kasaeian A, Khosravi B, Sorouri M, Azizi F, et al. Clinical characteristics and outcomes of diabetics hospitalized for covid-19 infection: A single-centered, retrospective, observational study. EXCLI J. 2020;19:1533-43. doi: 10.17179/excli2020-2988.

34. Besharat S, Alamdari NM, Dadashzadeh N, Talaie R, Mousavi SS, Barzegar A, et al. Clinical and demographic characteristics of patients with covid-19 who died in modarres hospital. Open Access Maced J Med Sci. 2020;8:144-9. doi: 10.3889/oamjms.2020.5013.

35. Rokni M, Ahmadikia K, Asghari S, Mashaei S, Hassanali F. Comparison of clinical, para-clinical and laboratory findings in survived and deceased patients with COVID-19: diagnostic role of inflammatory indications in determining the severity of illness. BMC Infect Dis. 2020;20:869. doi: 10.1186/s12879-020-05540-3.

36. Barkhordari K, Khajavi MR, Bagheri J, Nikkhah S, Shirzad $\mathrm{M}$, Barkhordari S, et al. Early respiratory outcomes following cardiac surgery in patients with COVID-19. J Card Surg. 2020;35:2479-85. doi: 10.1111/jocs.14915.

37. Ghaffari Rahbar M, Nafar M, Khoshdel A, Dalili N, Abrishami A, Firouzan A, et al. Low rate of COVID-19 pneumonia in kidney transplant recipients-A battle between infection and immune response? Transpl Infect
Dis. 2020;22:e13406. doi: 10.1111/tid.13406.

38. Monfared A, Dashti-Khavidaki S, Jafari R, Jafari A, Ramezanzade E, Lebadi MK, et al. Clinical characteristics and outcome of COVID-19 pneumonia in kidney transplant recipients in Razi hospital, Rasht, Iran. Transpl Infect Dis. 2020;22:e13420. doi: 10.1111/tid.13420.

39. Khwaja A. KDIGO clinical practice guidelines for acute kidney injury. Nephron Clin Pract. 2012;120:c179-c84. doi: 10.1159/000339789.

40. Yang X, Jin Y, Li R, Zhang Z, Sun R, Chen D. Prevalence and impact of acute renal impairment on COVID-19: A systematic review and meta-analysis. Crit Care. 2020;24: 356. doi: 10.1186/s13054-020-03065-4.

41. Robbins-Juarez SY, Qian L, King KL, Stevens JS, Husain SA, Radhakrishnan J, et al. Outcomes for Patients With COVID-19 and Acute Kidney Injury: A Systematic Review and Meta-Analysis. Kidney Int Rep. 2020;5:1149-60. doi: 10.1016/j.ekir.2020.06.013.

42. Who Coronavirus Disease (COVID-19) Dashboard. Available from: https://covid19.who.int/table.

43. Chan L, Chaudhary K, Saha A, Chauhan K, Vaid A, Zhao $\mathrm{S}$, et al. AKI in Hospitalized Patients with COVID-19. J Am Soc Nephrol. 2021;32:151. doi: 10.1681/ASN.2020050615.

44. Mohammadi Kebar S, Hosseini Nia S, Maleki N, Sharghi A, Sheshgelani A. The incidence rate, risk factors and clinical outcome of acute kidney injury in critical patients. Iran J Public Health. 2018;47:1717-24.

45. Momeni A, Ali FK, Behvandi B, Ganji F. Evaluation of prevalence and causes of acute kidney injury and its effect on short time mortality in intensive care unit patients. J Clin Diagn Res. 2017;11:OC10-2. doi: 10.7860/ JCDR/2017/21575.10722.

46. Samimagham HR, Kheirkhah S, Haghighi A, Najmi Z. Acute kidney injury in intensive care unit: incidence, risk factors and mortality rate. Saudi J Kidney Dis Transpl. 2011;22:464-70.

Copyright (C) 2021 The Author(s); Published by Nickan Research Institute. This is an open-access article distributed under the terms of the Creative Commons Attribution License (http://creativecommons.org/licenses/by/4.0), which permits unrestricted use, distribution, and reproduction in any medium, provided the original work is properly cited. 